\title{
Super-Humeanism: A starving ontology
}

\author{
Dustin Lazarovici* \\ University of Lausanne, Department of Philosophy
}

July 7, 2018

\begin{abstract}
The paper provides a critical discussion of the Super-Humean view of spacetime (Huggett's regularity account) and the "minimalist ontology" in terms of Leibnizian relations and primitive matter points, recently developed by Esfeld et al. It investigates, in particular, the empirical adequacy of the proposed metaphysics, arguing that Super-Humeanism cannot provide a plausible account of space and time without committing to bona fide geometric structure in the fundamental relations. Against this backdrop, I propose a moderate version of Super-Humeanism and discuss its possible application to Euclidean space and General Relativity.
\end{abstract}

"The appearances are a sight of the unseen."

- Anaxagoras, Fragment 21a

\section{The radicalization of Humeans}

Ever since its inception, which is widely attributed to the work of David Lewis (see e.g. Lewis 1973, $1986,1994)$, the program of Humean supervenience has grown bolder and larger in scope. While Lewis' account of laws of nature was mainly motivated by his rejection of modal connections, other authors have employed the best system strategy to ban all sorts of properties or entities, which they deem metaphysically suspicious, from the fundamental ontology of the world. Indeed, from the point of view of many modern Humeans, Lewis was too generous in outfitting the Humean mosaic, by admitting intrinsic qualities in addition to spatiotemporal relations. And while he discussed laws as regularities - the "theorems of the best system" (Lewis, 1994, p. 478) - the focus has shifted more towards the fundamental axioms from which these theorems are derived. The key to understanding the more recent and ambitious Humean programs is to appreciate the following two insights:

i) All empirical data can be ultimately understood as consisting in the distribution of matter in space and time (including pointer positions, display readings, computer printouts, or whatever else records the outcomes of experiments).

ii) While the strength of the best system candidates is measured against the regularities in the world, the laws of nature, as described by our best physical theories, are not just universal generalizations but involve all sorts of physical constants, dynamical variables, and geometric structures that figure in the mathematical formulation of laws of temporal evolution.

Hence, the Humean, if she is bold enough, can maintain that the Humean mosaic consists only in the spatiotemporal distribution of matter - made up by localized objects such as particles - while all other structures and quantities appearing in the formulation of physical theories are part of the best system, introduced to provide a simple and informative summary of the mosaic.

\footnotetext{
*Dustin.Lazarovici@unil.ch
} 
Structures and quantities that have thus been subject to some form of Humean reductionism include physical properties such as mass and charge (Hall, 2009, Esfeld et al., 2017), dynamical objects like the wave function in (Bohmian) quantum mechanics (e.g. Esfeld et al., 2014, Esfeld, 2014, Miller, 2014, Callender 2015, Bhogdal and Perry, 2017), and even geometric structures describing an absolute space or spacetime (Huggett, 2006, Vassallo and Esfeld, 2016). This is to say that the Humean can defend a relationalist conception of space (and time), without providing a relational reformulation of the physical laws, by maintaining that the absolute background space, presupposed by theories such as Newtonian mechanics or General Relativity, is merely a descriptive tool, allowing for a more efficient summary of the relational history of matter. The corresponding view is also known as Super-Humeanism, in analogy (and opposition) to super-substantivalism in the philosophy of spacetime.

Of course, not all Humeans go equally far in their reductionism. The best system strategy has proven very efficient in ridding the world of "non-Humean whatnots", but what is considered as a nonHumean whatnot, deserving of the Humean exorcism, varies from author to author. One of the most radical and comprehensive Super-Humean projects is due to Esfeld, Deckert and Vassallo (e.g. Vassallo et al., 2016, Esfeld and Deckert, 2017). The aim of this project is to spell out an ontology of the natural world that is as parsimonious as possible, while being overall coherent and empirically adequate. The proposed ontology consists in primitive matter points, without intrinsic properties, individuated by primitive distance relations (axiom 1). These matter points are permanent, with the distances between them changing (axiom 2).

All that exists in the world is thus a network of changing distance relations between bare matter points. The role of physics is now to provide the most efficient description of the history of changing distance relations - striking an optimal balance between simplicity and strength - by introducing appropriate dynamical parameters and geometric representations. In a recent book (Esfeld and Deckert, 2017), this view is spelled out in detail and applied to a wide range of modern physical theories from Newtonian mechanics, to Bohmian quantum mechanics, to general relativity and even quantum field theory, where the authors show how an ontology of permanent point particles can account for the phenomena appearing as "particle creation and annihilation" (cf. Deckert et al., 2017).

It is important to note that the distance relations postulated by Esfeld and collaborators are directionless, dimensionless and structureless. They are constrained only by the triangle inequality which is arguably the minimal requirement to make them distance relations that hold together the material world (in contrast to, let's say, hypothetical thinking relations that hold together a world of minds), but there is nothing about the relations that would make them 3 or 2 or 10-dimensional, Euclidean or non-Euclidean distances. This is in notable contrast to the relational project championed, in particular, by Julian Barbour (see e.g. Barbour and Bertotti, 1982), whose fundamental relations carry much more geometric structure (precisely the non-absolute Euclidean structure in the non-relativistic case) and are meant to constrain the dynamical laws, in the sense that the formulation of the laws should not refer to more or different geometric structure than what is provided by these fundamental relations. Upon the Super-Humean strategy, first proposed by Nick Huggett (2006) and adopted by Esfeld et al., all geometric features have been delegated to the best system. The Humean mosaic consists only in structureless distance relations between point particles (primitive matter points), while the candidates for the best system description vary not only with respect to the dynamical laws but also with respect to the spatiotemporal geometry used to represent the history of distance relations and formulate the laws of motion. Hence, from the fundamental point of view, all geometric structures are nothing more than mathematical or representational surplus. Yet, of all the representational surplus that one could posit, one 
combination (the Super-Humean hopes) will be "true" in virtue of striking the optimal balance between simplicity and strength. In this sense, the (apparent) geometry of space or spacetime supervenes, together with the dynamical laws, on a purely relational, non-modal and non-geometric ontology.

We should pause for a second to appreciate the implications of this view. It is to say that space appears to be 3-dimensional, not because geometric relations instantiated in the world are 3dimensional, but because the contingent history of distance relations happens to be such that it can be most efficiently summarized in a 3-dimensional representation. It is also to say, for instance, that when Einstein presented his theory of relativity, he didn't discover anything new about the nature of space and time, but rather observed that the history of distance relations can be more efficiently summarized in a 4-dimensional, Lorentzian spacetime geometry. Ultimately, according to the view of Esfeld, Vassallo and Deckert, physics seems neither capable nor in charge of informing us about the fundamental ontology of the world. Since they have established that a) all empirical facts can be understood as facts about the configuration of matter and b) facts about the configuration of matter can be conceived as facts about distance relations between primitive matter points, it is unclear what empirical evidence or scientific discoveries would compel them to revise their ontology.

\section{One trick pony}

While this proposal is very radical, even by Humean standards, I believe it can be seen as a more or less natural consequence of the Humean program. Certainly, in actual scientific practice, the fundamental ontology of physics is not fixed a priori; instead, different theories vary not only with respect to their laws, but also with respect to the fundamental entities (or natural properties in the sense of Lewis) that these laws are referring to. In Humean terms, this is to say that different candidates for the best system provide not just competing summaries of the same Humean mosaic but may describe a completely different outfit of the mosaic to begin with. Laws and ontology come as a "package deal" (Lower 2007).

However, under this conception, there seems to be a tension between the ideas that a) the physical ontology is theory-dependent, coming in a package with the physical laws, and b) there are "good" standards to compare these packages, which compete for the best system, with respect to their strength as summaries of the mosaic.

"Good standards", I think, are those expressed by Hall (2009) through the metaphor of "Limited Oracular Perfect Physicist" [LOPP]. They should be objective enough, so that a perfectly rational physicist, equipped with sufficient knowledge about the physical facts throughout the history of the universe, would come to an unambiguous judgement about what the best system is, though not require metaphysical omniscience like knowing "the laws of nature" or "the true ontology of the world" as a primitive fact. To emphasize: the worry here is not that different theories, postulating a different fundamental ontology, would tie for the best system but that they would be incommensurable along the dimension of "strength". As Hall (2009) writes (contra Loewer, 2007):

Put in terms of our LOPP, the worry is this: She needs to be given some information about the world - information that is specifiable independently of what the best system is - in order to have a basis on which to judge the various law-hypotheses.

In conclusion, there is a certain pressure on the Humean to make a priori ontological commitments before engaging in negotiations about the best system. That is to say positive commitments about what there is in the world, in addition to the usual negative theology about necessary connections, etc. Lewis, of course, had made some such commitments; Esfeld and Deckert take it a step - or 
maybe a few steps - further, by essentially trying to fix the ontology of physics once and for all. We must concede that this approach provides a viable solution to the above-mentioned dilemma: if the physical ontology is not theory-dependent, but fixed on the basis of a priori metaphysical considerations, all candidates for the best system will refer to the same fundamental ontology -i.e. the same mosaic - and can compete in terms of strength and logical simplicity.

On the other hand, I believe that by succumbing to the idol of parsimony and taking Humean reductionism to this extreme, Esfeld et al. also demonstrate that Super-Humeanism as an a priori thesis is both uninteresting and uncompelling since it is too promiscuous and universally applicable. When all is said and done, there is one and only one line of reasoning that is used to ground successful physical theories in her preferred ontology. I call it the Super-Humean subterfuge:

Our physical description of the world exhibits the feature $X$ because the contingent relational distribution of matter throughout the history of the universe happens to be such that the best system description exhibits the feature $X$.

As noted before, the problem with this reasoning is not that it couldn't be coherently defended, but that the general arguments put forward in defense of Super-Humeanism and the minimalist ontology of Esfeld and Deckert would seem to apply independent of what X stands for. If every part of a physical theory can be delegated to the best system mythology, no part needs to affect your ontological commitments.

Notably, the claim here is not that a priori everything could supervene on regularities in the relational distribution of matter. Qualia or normative facts, for instance, may not - but they are also not within the scope of any physical theory. The basic idea underlying Esfeld's project is rather that a Humean mosaic made up of relational configurations of matter is sufficient to ground all empirical facts that are the target of naturalistic accounts; and that any other structure appearing in the formulation of a physical theory can then be understood through its role in describing or summarizing such a mosaic (or else it is superfluous). For instance, a physical account of color perception can be given in terms of particles moving in our brain and visual receptors - in a way that is correlated with particle motion in the perceived objects - and the regularities of these particle motions can be described by means of an electromagnetic field. But neither colors nor electromagnetic fields then need to be admitted into the fundamental ontology of the world. (Many Humeans will admit at least the latter, but Esfeld and Deckert do not.)

It will not be argued in this paper that this strategy is always successful (ultimately, I hope to convince the reader that it is not, though I would insist that it is at least logically consistent). My point here is rather that if we accept the strategy as successful with respect to electromagnetic fields, and quantum wave functions, and spacetime structures, it is hard to see how it could ever fail. Whether physics postulates new fundamental kinds, or introduces new dynamical structures, or changes its understanding of space and time, it would always seem possible to adopt these innovations as mere descriptive tools, introduced to provide a more efficient summary of the Humean mosaic, without revising our opinions about what constitutes the mosaic in the first place. To sum up in more polemical terms: The Super-Humean is a bit of a one trick pony and the trick is starting to get old already.

Esfeld and Deckert, very much to their credit, defend various alternatives to established theories that are more compatible with an ontology of permanent matter points. In grounding physical theories in their minimalist ontology, they are willing to accept as much mathematical surplus structure as necessary, but they clearly prefer less to more surplus, if possible. Still, since it is impossible to provide a simple and empirically adequate description of the world solely in terms of structureless distance relations, they invariably have to fall back on the subterfuge sooner or later. 
The metaphysics of Esfeld et al. is committed to naturalism (or even physicalism) but it is not, despite their claims, naturalized metaphysics in the sense of being guided or informed, in any significant way, by our best scientific theories. This may seem like a mere methodological critique, unless we already took for granted that naturalized metaphysics is good and all other metaphysics is bad. However, a reasonable standard for rejecting their metaphysical proposal cannot be to show that it is strictly impossible or inconceivable as an ontology of the natural world (I already granted that it is not). Instead, focusing on Super-Humeanism in the narrower sense, as an interpretation of space or spacetime, I am going to argue that structureless distance relations are not impossible but implausible as a fundamental structure grounding a 3-dimensional geometry of space, respectively a 4-dimensional geometry of spacetime, to the extent that a Super-Humean ontology cannot be considered empirically adequate by reasonable standards.

\section{Space, lost}

The Super-Humean strategy to ground physical theories in a minimal relational ontology was laid out by Nick Huggett (2006) in his relationalist (re)interpretation of Newtonian mechanics. This will also serve as the test-case for our following discussion. While Newtonian mechanics is no longer considered a fundamental theory, it is still the preferred playground of many philosophers; and while it may not provide the best system description of the world, it is still a very good and useful one. A possible extension of Huggett's regularity account to general relativistic spacetime is discussed in Esfeld and Deckert (2017, ch. 5) as well as in Vassallo and Esfeld (2016). By and large, it is just an adamant application of the Super-Humean subterfuge; the following objections will carry over accordingly. (In fact, the general relativistic case fares somewhat better in that curved spacetime yields more freedom in embedding the matter points. It fares worse in that the history of co-present configurations cannot be recovered from a description on relativistic spacetime, which has no structure of absolute simultaneity. Hence, it is unclear in what sense the alleged best system even makes true statements about the mosaic.)

I want to emphasize that my objections are targeted against the full-fledged Super-Humeanism spelled out by Huggett and adopted by Esfeld et al. The aim of their regularity theory is essentially two-fold (the second being an escalation of the first):

1. To ground physical theories referring to an absolute background space or spacetime in a relationalist ontology without providing a relational reformulation of the physical laws.

2. To reduce all geometric features of this background space - its dimension, symmetries, curvature etc. - to thin distance relations that carry no a priori geometric structure.

These two points are not entirely independent: the more structure the fundamental relations carry, the less flexible will the Super-Humean ontology be in accommodating different background spaces described by different theories. Still, in principle, a not-quite-so-super Humean could let go of the second aim, while still invoking Huggett's regularity theory to accomplish the first. i.e. postulate thicker spatial or spatio-temporal relations as the supervenience base of the absolutist physical description. (I don't want to go into a deep metaphysical discussion about thin versus thick relations; I am merely using the terminology to distinguish between the minimalistic relations of Huggett and Esfeld, that are constraint only by the triangle inequality, and other relational accounts in which the fundamental relations carry more geometric structure, e.g. a 3-dimensional direction in addition to a distance). We will explore this option in the next section. Our following discussion, however, is concerned with point 2, that is, with the Super-Humean claim to get away without committing to bona fide geometric structures in her fundamental ontology. In other words, the conflict here is not between relationalism and substantivalism, but between a geometric and a non-geometric ontology, 
making the substantivalist, the spacetime structuralist and even the liberal relationalist à la Barbour or Saunders (2013) unlikely allies against the Super-Humean minimalism.

To make precise what the Super-Humean relations are, we recall the definition of Esfeld and Deckert (2017) who specify the relational structure instantiated by $N$ matter points by the following set of axioms. (For convenience, the matter points are labeled by an index $i \in\{1, \ldots, N\}$; the indices are, however, arbitrary and not supposed to indicate a primitive identity of the matter points.)

i) Any two matter points stand in a distance relation that can be represented by a positive real number $r_{i j} \in \mathbb{R}^{+}, 1 \leq i \neq j \leq N$.

ii) The relation is symmetric, i.e. $r_{i j}=r_{j i}, \forall 1 \leq i \neq j \leq N$.

iii) The numerical assignments satisfy the triangle inequality: $r_{i k} \leq r_{i j}+r_{j k}, \forall i, j, k \leq N$.

Esfeld and Deckert also require that the matter points are individuated by the distance relations (thereby insisting on what has become known as absolute discernibility). That is, the matter points have no intrinsic identity, but any two points are numerically distinct by virtue of standing in a different relation to at least one other matter point. To this end, the authors must add a fourth postulate, excluding symmetric configurations in which some particles would no longer be absolutely discernible. While this combination of relationalism and moderate ontic structural realism comes with its own set of interesting problems (most notably: what provides for the identity of the matter points over time, as the configuration of distance relations changes?), those are beyond the scope of this paper. What matters for our present discussion is that the above axioms are very weak, essentially emulating the general mathematical definition of a "metric". To repeat: fundamentally, there is nothing about the distance relations that would make them 3-dimensional or Euclidean, or put any other constraints on the dimension, curvature or topology of the physical geometry (Huggett, 2006, clearly agrees with this point).

According to the Super-Humean regularity theory, the fact that Newtonian mechanics is formulated on a 3-dimensional Euclidean space only means that this geometric representation - together with the laws of Newtonian mechanics - strikes a good balance between simplicity and strength in summarizing the history of structureless distance relations. The candidates for the best system thereby vary not only with respect to the dynamical laws, but also with respect to the background space (qua mathematical structure) used to represent or embed the history of distance relations in the first place. Such an embedding has to preserve only the distances between the matter points, since there is no other structure to preserve. That is, if we denote the history of distance relations by $r_{i j}(\lambda)$, with $\lambda$ some arbitrary parameter, the representation of the matter points as trajectories $q_{i}(\lambda)$ on some metric space $(M, d)$ must satisfy $d\left(q_{i}(\lambda), q_{j}(\lambda)\right)=r_{i j}(\lambda)$. All other things being equal, a lower-dimensional space $M$ and higher degree of symmetry are preferred on grounds of simplicity (Huggett, 2006, p. 54), but the fact that space appears to be 3-dimensional, and at least locally Euclidean, is purely contingent according to the Super-Humean account.

I strongly agree with Belot (2000, p. 10) that such an interpretation "is arrant knavery: a cheap instrumentalist rip-off of Newtonian theory", but I doubt that one can shame the Super-Humean into yielding any ground. Instead, I want to make the case that the Super-Humean reduction of Euclidean space is simply unsuccessful by reasonable standards. What the account fails to acknowledge, is that it is no trivial matter to embed such a relational network into 3-dimensional Euclidean space. It is always possible to embed a triplet of particles: the result is simply a triangle (or a line, in the degenerate case) whose side-lengths correspond to the distances between the matter points. The triangle inequality - which the distance relation must satisfy by definition - is sufficient to ensure the existence of such an embedding. However, in order to embed four, or five, or six matter points, 
additional constraints on the distance relations would have to be satisfied, which would be purely accidental upon the Super-Humean view. A similar observation was made by Maudlin (2007, pp. 8789), who argues that the substantivalist, but not the relationalist, can explain such constraints, in particular the basic triangle inequality that the relationalist has to accept as an axiom (while the substantivalist can derive it from the concept of "path lengths"). I am not concerned about the status of the triangle inequality but want to elaborate on the case of multiple particles, that is, on the additional constraints that are not even axioms but mere contingencies upon the Super-Humean account. On this basis, I will defend the thesis that the most basic empirical fact about space, namely its low dimensionality, is not just unexplained by the regularity theory but should be regarded as a falsifying instance.

To illustrate why the embedding on multiple matter points into 3-dimensional Euclidean space requires additional constraints, assume we got lucky and were able to embed four matter points while preserving their mutual distances. Embedding a fifth matter point now corresponds, pictorially speaking, to determining the point of intersection of four spheres in 3d-space (each centered around one of the existing matter points with radius equal to its distance to the fifth). However, four spheres in 3-dimensional Euclidean space do not have a point of intersection, unless their radii happen to satisfy additional algebraic relations. In general, we would have to move into 4-dimensional space to faithfully represent the distance relations between all five matter points. And the larger the network of distance relations, the more (and more specific) constraints on the relations must be satisfied to fit a given configuration into a 3-dimensional geometry.

More precisely, embedding $N$ points into $d$-dimensional Euclidean space amounts to assigning $d(N-1)$ coordinates. (Without loss of generality we can fix one particle to the origin of the coordinate system, leaving $N-1$ points with $d$ coordinates each. In fact, accounting for global rotations, we have only $d(N-1)-\frac{1}{2} d(d-1)$ relational degrees of freedom - and one less if we factor out absolute scale -, but these corrections are negligible for the following estimates.) On the other hand, there are $\left(\begin{array}{l}N \\ 2\end{array}\right)=\frac{1}{2} N(N-1)$ distance relations between $N$ matter points, amounting to the same number of quadratic equations for the coordinates as we want to realize the relations as Euclidean distances on $\mathbb{R}^{d}$. (The coordinates of two points $x, y \in \mathbb{R}^{d}$ with distance $r$ must satisfy the quadratic equation $\left(x_{1}-y_{1}\right)^{2}+\cdots+\left(x_{d}-y_{d}\right)^{2}=r^{2}$.) In conclusion, as soon as $N>2 d$, the system is overdetermined and will not admit any solution at all unless roughly $\frac{1}{2} N(N-1)-$ $d(N-1)=\left(\frac{1}{2} N-d\right)(N-1) \approx \frac{1}{2} N^{2}$ additional constraints are satisfied.

For our universe, $N$ is estimated to be about $10^{80}$. Considering only the visible matter in our most immediate surrounding - allowing for the far-fetched possibility that space doesn't look 3dimensional everywhere - the number of particles is still at least of the order of $10^{35}$. Hence, the number of geometric constraints that would have to be satisfied - purely accidentally - for a network of Super-Humean relations to be embeddable into 3-dimensional Euclidean space is at least of the order $10^{70}$. Yet, the Super-Humeans ask us to believe that this epic coincidence obtains not only within one particular configuration, but that the trillions of trillions of trillions of constraints happen to be preserved over time, as the distance relations between the matter points change. Mind you, according to their metaphysics, there is nothing in the world that would make it necessary for these constraints to be satisfied in any configuration, let alone propagate with the dynamics.

Calling this proposal "implausible" would be a gross understatement. In physics, we would never accept a theory that requires $10^{70}$ fine-tuned parameters to explain even the most basic empirical facts. I don't see why metaphysical theories should be held to that much lower standards. To put it the other way around: by assuming a 3-dimensional space in which the matter points move - that is, 
a real physical space that constraints their motion, not just a mathematical construct that describes it - we explain at least $10^{70}$ dynamical constraints that the Super-Humean accepts as bare facts. This is a successful reductive explanation if there ever was one.

I imagine a Super-Humean response to go somewhat like this: Admittedly, we cannot give a deeper metaphysical explanation for why the history of distance relations is such that it can be represented on a 3-dimensional Euclidean space. But you, as a "substantivalist", cannot give any deeper metaphysical explanation for why space is 3-dimensional (and at least locally Euclidean), rather than 4 or 5 or 17,000 dimensional. Of course, the 3 -dimensional representation of the world contains a lot of information about the history of distance relations, but this is precisely what makes this geometry part of the best system. In other words, all those algebraic relations that hold between the particle distances are part of the regularities that we find in the world, and they are summarized in our 3dimensional representation of the (Newtonian) laws, which thus provides the kind of unifying explanation that we should expect from science.

This Humean response has some persuasive power, but it is based on a completely false equivalence. Obviously, a substantivalist or a liberal relationalist (who admits thicker relations as fundamental) postulates an absolute space, respectively some geometric structure, as a physical and metaphysical primitive. She thereby accepts certain facts about the geometry of the world as primitive, such as the fact that space has 3 dimensions rather than 4 or 5 or 17,000. But every theory needs some primitives; and the geometry of space or spacetime is arguably so basic to our conception of the world, and so informative about the possible configurations of matter in it, that it seems like a reasonable choice. The Super-Humean doesn't deny that space or spacetime are real in some sense that there are true geometric facts about the world - but she claims to provide a reductive account of these facts. It is she who must therefore deliver on her promises. The Super-Humean reduction, however, fails since it has to assume an exceedingly special configuration of distance relations throughout the history of the universe to account for even the most basic geometric facts.

One reason why we should not accept such an account as successful is that it "reduces" a small number of simple primitive facts to a huge number of complicated primitive facts. One reason why we cannot accept such an account as successful is that we would give up on any means to test a theory about what exists in the world against any facts that we could possibly know about the world if we admitted that the actual world need not look anything like a typical one that the ontology can possibly constitute.

From this point of view, I claim that the Super-Humean account of space is not just explanatory deficient but empirically inadequate. Remember that when embedding $N$ points in a $d$-dimensional Euclidean space, we have the freedom to choose $d(N-1)$ coordinates, while having to solve for $\frac{1}{2} N(N-1)$ quadratic equations, expressing the distance relations that the points bare to one another. This means that given a typical set of distance relations, the dimension of the lowestdimensional space into which it can be embedded will be of the order $d \approx \frac{1}{2} N$, with $N \approx 10^{80}$ ! In this sense, the metaphysical theory of Super-Humeanism makes a prediction, namely that space should look extremely high-dimensional, if it has any meaningful geometric structure at all. Since the opposite is true, and we seem to live in 3-dimensional space or 4-dimensional spacetime (even the 10 or 11 spaces required by string theory would count as very low-dimensional in this context), we must conclude that a Super-Humean ontology of structureless distance relations doesn't fit the world that we experience.

Notably, a similar objection could be raised against Humeanism in general, that is, against the regularity view of laws, since a typical Humean world would arguably not exhibit the kind of simple 
and reliable regularities that we observe in nature. I believe that this argument is successful and that Humeanism is empirically inadequate in general. I also believe, however, that the Super-Humean is in an even worse position with respect to space or spacetime than with respect to dynamical laws. The regular Humean can at least with some credibility carry the banner of empiricism. She can argue that she and her opponent agree on all concrete physical facts in the world and disagree only about the status of modality. And she can further make the case that, since we don't have any direct empirical access to modal connections, we should go with the deflationary, i.e. more parsimonious account. The Super-Humean and her opponent - be it the substantivalist or the liberal relationalist à la Barbour - do not agree on the concrete physical facts in the world. For her opponents, the spatial relations instantiated in the world (be it fundamentally between particles or between particles in virtue of occupying certain points in space) carry more geometric structure, characterized not only in terms of (relative) distances but also in terms of 3-dimensional directions. And it is these thick relations - not the thin relations - to which we have the most direct empirical access. We experience objects as having a shape and a location - not just a distance - relative to one another. Hence, even the Super-Humean must admit that it is the 3-dimensional representation, not the unordered list of primitive distance relations, that has the most intimate connection to our manifest image of the world. And then the Super-Humean can call her relations "spatial" all she wants. What she has in her ontology is not space, as commonly understood and experienced, but a much more impoverished notion; starving thin relations that can only emulate spatial relations for the price of assuming an utterly atypical, meticulously fine-tuned configuration throughout the history of the universe.

To sum up more systematically, let us return to the promiscuousness of the Super-Humean subterfuge discussed in section 2 . That a proposal for a fundamental ontology of the natural world can in principle fit our experience and scientific description is an extremely low bar that saves us neither from arbitrariness nor from absurdity. In principle, one can postulate any ontology, provided only that it affords enough degrees of freedom to tweak, and assume that these degrees of freedom are arranged in precisely such a way as to instantiate (or be in some sense isomorphic to) whatever structures identified in nature. The question we must ask is therefore: what distinguishes a serious and plausible candidate for ontology from a mere metaphysical prejudice? Or, in other words: what distinguishes a legitimate application of the subterfuge from one that is trivial, spurious and ad hoc? A few possible criteria come to mind, and Super-Humeanism fails all of them:

1. We can require that the fundamental ontology matches in some sense the structures or objects appearing in the formulation of our best physical theories. This criterion - marking the still dominant methodology in naturalized metaphysics - is explicitly rejected by SuperHumeans in general and Esfeld et al. in particular. Hence, it is not surprising that their account fails in this respect: no successful physical theory is formulated in terms of structureless distance relations between bare matter points, and arguably none ever will be.

2. We can require that our actual world resembles not just a particularly special and fine-tuned model of our metaphysical theory but a typical one. Humeans in general seem to reject this criterion and Super-Humeanism fails it in a particularly spectacular fashion since a typical world formed and held together by structureless distance relations would not look anything like a material world in a low-dimensional space or spacetime.

Note, on the other hand, that for a substantivalist, the apparent truism that "space looks 3dimensional because it actually is 3-dimensional" involves just such a typicality reasoning. It is possible - though highly atypical - in a 3-dimensional Newtonian universe that all particles move on a 2-dimensional hyperplane. Such a world would appear 2-dimensional to the hypothetical flatlanders living in it. It is equally conceivable and physically possible that we are 3-dimensional "flatlanders" living in a higher-dimensional Euclidean space. The postulate 
of a 3-dimensional space matches the appearance of a 3-dimensional space because it makes the latter typical.

3. We can require that the connection between ontology and experience is in some sense direct, simple and robust. In other words, this is to insist on a reasonably short cognitive distance between our manifest and scientific image of the world. (The terms "manifest image" and "scientific image" go back to Sellars, 1963; for an interesting discussion along these lines, see Maudlin, 1997). Some version of this criterion is in fact the main argument of so-called primitive ontology theories, which postulate a local ontology in 3-dimensional space or 4-dimensional spacetime, against functionalist approaches such as wave-function realism that try to connect our experience of the world to a more abstract description of physical reality by some sort of functionalist emergence. Esfeld and collaborators attempt, at times, to carry the banner of the primitive ontology program. Their terminology can easily suggest that the "Leibnizian distance relations" are just the intuitive spatial relations that we perceive between macroscopic objects, and that the objects themselves can be straightforwardly conceived of as a collection of primitive matter points. For the reasons just discussed, this would be quite misleading. The connection between the network of distance relations and our experience of the material world is not direct. It is provided in terms of best system representation of the history of distance relations, not in terms of the fundamental ontology. The connection is not simple. It must take the detour of the best system account, a highly complex procedure of trying and comparing different summaries and representations of the entire history of the universe. And the connection is not robust. It relies on an extremely special and fine-tuned arrangement of the Humean mosaic, and even a small perturbation of this arrangement would lead to a radically different appearance of the physical world.

The criteria for ontology that Esfeld et al. announce to follow is "parsimony - together with empirical adequacy". However, as we have just seen, the Super-Humean account is empirically adequate only by standards that render the criterion itself trivial; that is, in the sense in which any ontology could be postulated as the supervenience base of our scientific description of the world if it is just sufficiently complex and sufficiently fine-tuned, and if the supervenience relations are sufficiently indulgent (as Humean supervenience is). Which leaves us with parsimony as the only success of their "minimalist ontology".

This is not good enough. Humeans who are guided by a principle of parsimony seem to believe that postulates of what there is in the world are costly, while assumption about how it is come for free. But the two issues cannot be disentangled, since we cannot pass rational judgement on one without the other. Parsimony may be a good criterion for the what question, but we need additional criteria, such as the ones proposed above, to judge it in conjunction with a how hypothesis. To postulate a parsimonious ontology, and then assume that this ontology is arranged however it needs to be to get the phenomena right, is trivial and doesn't lend any credibility to the ontological claims. And to delegate the how questions entirely to physics and empirical science, which should inform us how the fundamental entities are arranged and how they evolve in time, doesn't work either, unless we take our ontological commitments from science, as well. Physics per se doesn't inform us about Super-Humean relations because no established physical theory is about Super-Humean relations. And even if it did, it would do so in terms of geometrical and dynamical structures that are equally if not more fundamental in the physical theory. If we insist on the autonomy of metaphysics to determine the fundamental ontology of the world, and what our best physical theories are actually referring to, we cannot get too comfortable in our armchairs. Good practice, both in science and metaphysics, is not to multiply fundamental entities, properties or structures beyond necessity. But it is certainly a necessity for a theory to match, in some serious sense, the world that we experience. 


\section{A moderate Super-Humeanism}

Our previous discussion leaves open the possibility of thickening the fundamental spatial relations, while still applying the regularity theory to ground our most successful physical theories in a relationalist ontology. In particular, departing from the strict minimalism of Huggett and Esfeld, one could postulate fundamental spatial relations that carry both a distance and a (relative) 3dimensional direction. Mathematically, such a 3-dimensional directed distance relation between matter points $i, j \in\{1, \ldots, N\}$ would be represented by a 3-dimensional vector $\boldsymbol{r}_{i j} \in \mathbb{R}^{3}$, rather than a single positive number. More precisely, to have a proper relational structure, we should understand the set $\left\{\boldsymbol{r}_{i j} \in \mathbb{R}^{3} \mid i \neq j=1, \ldots, N\right\}$ modulo a global rotation (and possibly modulo a global scale factor). That is, we care only about the relative orientation (and lengths) of the distance vectors. In any case, this directed distance relation would be antisymmetric rather than symmetric, i.e., $\boldsymbol{r}_{i j}=$ $-\boldsymbol{r}_{j i}$. And it would be constraint by the composition rule $\boldsymbol{r}_{i j}=\boldsymbol{r}_{k j}-\boldsymbol{r}_{k i}$, which implies the triangle inequality for the distances (when considering the norm of the vectors, $\left\|\boldsymbol{r}_{i j}\right\|=\left\|\boldsymbol{r}_{k j}-\boldsymbol{r}_{k i}\right\| \leq$ $\left.\left\|\boldsymbol{r}_{k j}\right\|+\left\|\boldsymbol{r}_{k i}\right\|\right)$ but is much more restrictive. It leaves us with exactly $3(N-1)$ degrees of freedom, before factoring out global scale and orientation, compared to the $\frac{1}{2} N(N-1)$ degrees of freedom in the minimalist relational network of Esfeld et al.

Such a "thick" relational configuration can always be embedded into 3-dimensional Euclidean space (e.g. fix a coordinate system, place matter point 1 at the origin and matter point $j$ at $\boldsymbol{r}_{1 j} \in \mathbb{R}^{3}$ ), thus avoiding the objections raised in the previous section. The embedding is even unique - modulo global rotation, translation, and possibly rescaling - since these directed distances capture precisely the relational structure of 3-dimensional Euclidean space.

Notably, the moderate Super-Humean is thereby not committed to dynamical laws formulated on shape space (as in the framework of Barbour and Bertotti, 1982), nor to the even weaker relationalism of Saunders (2013), which admits a primitive notion of parallelism between directed distances at different times, thus accounting for absolute rotations. As discussed in detail by Esfeld and Deckert (2017), it is consistent to maintain that the best system representation employs an absolute spatiotemporal geometry - qua mathematical structure - if this allows for a simpler and empirically adequate formulation of the dynamical laws. In this way, the authors also deal with rotating universes, without trying to provide a relational account of absolute rotations.

This moderate Super-Humeanism can be extended to general relativistic spacetimes along the lines of Esfeld and Vassallo (2016). The main challenge here is that in curved space, something akin to distance vectors can be defined only locally at a point (more precisely, in terms of tangent vectors to curves passing through that point). In particular, the spacetime metric defines angles only between vectors in the same tangent space (the set of all tangent vectors at a given point $x$ ), not between tangent vectors at different points. Fortunately, general relativistic spacetimes are described as Riemannian manifolds that come equipped with an additional structure - a connection - that allows us to identify tangent vectors at different points of the manifold by so-called parallel transport. In benign cases, this will allow us to represent our relational structure of 3-dimensional directed distances on a curved spacetime. To specify what these "benign" cases are, and carry out our moderate Super-Humean strategy, we have to impose two restrictions on the class of spacetime models.

The first restriction is that the relevant spacetime geometries be globally hyperbolic. Simply put, this means that our spacetime can be described as an ordered stack of 3-geometries on which we can represent the history of changing distance relations. In more technical terms, global hyperbolicity implies that the 4-dimensional spacetime manifold allows for a global time function and a foliation 
into 3-dimensional spacelike (Cauchy) hypersurfaces (see e.g. Wald, 1983, Thm. 8.3.14. See Callender, 2017 for a recent philosophical discussion).

The second assumption is that the leaves of the foliation (i.e. the 3-geometries) are geodesically complete. This implies, in particular, that any two points $x, y$ on such a spacelike hypersurface $\Sigma$ are connected by a unique geodesic (a path of minimal length). The length of the geodesic then corresponds to the distance between $x$ and $y$ on $\Sigma$, and there is even a well-defined "direction" given by a vector $\boldsymbol{X}$ in the tangent space $T_{x} \Sigma$ over $x$ - in which the geodesic path from $x$ to $y$ departs. Mathematically, the relevant concept is the so-called exponential map. It maps a tangent vector $\boldsymbol{X} \in T_{x} \Sigma$ to the end-point of the geodesic curve starting at $x$ in the direction of $\boldsymbol{X}$ with length $\|\boldsymbol{X}\|$. (The length of the vector being defined in terms of the space-time metric projected onto $T \Sigma$.) Thus, if $\exp _{x}(\boldsymbol{X})=y$, it makes sense to say that the tangent vector $\boldsymbol{X}$ represents the distance and direction in which $y$ lies from $x$.

Another mathematical concept we need to introduce is the above-mentioned parallel transport, connecting tangent spaces at different points of the manifold. A parallel transport between two points $x$ and $y$ carries a vector in the tangent space $X \in T_{x} \Sigma$ at $x$ to a vector in the tangent space $T_{y} \Sigma$ at $y$. On a curved space, the result of a parallel transport will in general depend on the path along which the vector is transported. However, since our hypersurfaces $\Sigma$ are geodesically complete, we can always perform the parallel transport along the unique geodesic connecting $x$ to $y$. This yields a well-defined identification of tangent spaces at different points of the manifold. For $x, y \in \Sigma$, we denote the corresponding parallel transport map by $P_{x \rightarrow y}: T_{x} \Sigma \rightarrow T_{y} \Sigma$.

Now, given a configuration of directed distance relations $\left\{\boldsymbol{r}_{i j} \in \mathbb{R}^{3} \mid i \neq j=1, \ldots, N\right\}$, we say that these relations are faithfully instantiated, or represented, by $N$ points $x_{1}, x_{2}, \ldots, x_{N} \in \Sigma$ if

(1) $\quad \exp _{x_{1}}\left(\boldsymbol{r}_{1 j}\right)=x_{j} ; \quad \exp _{x_{i}}\left(P_{x_{1} \rightarrow x_{i}} \boldsymbol{r}_{i j}\right)=x_{j}$,

where $\boldsymbol{r}_{i j} \in T_{x_{1}} \Sigma$ are understood in terms of a local orthonormal frame (so that the coordinate representation of the metric tensor corresponds to the Euclidean metric). The intuition is as follows: move from point $x_{1}$ in the direction $\boldsymbol{r}_{1 j}$, along the corresponding geodesic, and you reach $x_{j}$. Consider the vector corresponding to $\boldsymbol{r}_{i j}$ at $x_{i}$, move in the respective direction along the geodesic path, and you reach $x_{j}$. Then, we can say that the relational structure is instantiated by the points $x_{1}, \ldots, x_{N}$ on the 3-dimensional hypersurface $\Sigma$.

It is important to note that while (1) seems to privilege the point $x_{1}$, the condition is actually independent of the "base point" in the following sense: If for fixed $k \in\{1, \ldots, N\}$ we define $\overline{\boldsymbol{r}}_{i j}:=$ $P_{x_{1} \rightarrow x_{k}} \boldsymbol{r}_{i j}$, the set $\left\{\overline{\boldsymbol{r}}_{i j} \mid i \neq j=1, \ldots, N\right\} \subset T_{x_{k}} \Sigma$ will represent the same distance relations, since parallel transport with respect to the metric connection of general relativity (and its orthogonal projection onto $T \Sigma$ ) is orthogonal, i.e. preserves the lengths of the vectors and the angles between them. Moreover, due to the linearity of the parallel transport, the composition rule is still satisfied:

$$
\overline{\boldsymbol{r}}_{i j}=P_{x_{1} \rightarrow x_{k}} \boldsymbol{r}_{i j}=P_{x_{1} \rightarrow x_{k}}\left(\boldsymbol{r}_{k j}-\boldsymbol{r}_{k i}\right)=P_{x_{1} \rightarrow x_{k}} \boldsymbol{r}_{k j}-P_{x_{1} \rightarrow x_{k}} \boldsymbol{r}_{k i}=\overline{\boldsymbol{r}}_{k j}-\overline{\boldsymbol{r}}_{k i} \text {. }
$$

Now, it is straightforward to check that if the embedding $\left\{x_{1}, x_{2}, \ldots, x_{N}\right\} \subset \sum$ of matter points satisfies (1), it will also satisfy

$$
\exp _{x_{\mathrm{k}}}\left(\overline{\boldsymbol{r}}_{k j}\right)=x_{j} ; \quad \exp _{x_{i}}\left(P_{x_{k} \rightarrow x_{i}} \overline{\boldsymbol{r}}_{i j}\right)=x_{j}
$$

in which the base-point $x_{1}$ is replaced by $x_{k}$. In the upshot, the configuration of directed distance relations must first and foremost be understood in terms of tangent vectors at one of the embedded 
points, but parallel transport allows us to identify tangent spaces at different points of the 3dimensional hypersurfaces in a way that preserves the relational structure.

As already mentioned, one problem with this strategy is that it must exclude several classes of spacetime models that could be physically relevant. The assumption of global hyperbolicity excludes, in particular, spacetimes with closed time-like curves (CTCs), like the infamous Gödel universe or rotating black holes, while the assumption of geodesic completeness (of the sheets of foliation, not the entire manifold) excludes time-like singularities (though not the cosmological singularity at the big bang or a static black hole). Vacuum solutions, of course, must also be regarded as (meta)physically meaningless if spacetime geometry is only there to describe the history of matter points. I suppose the moderate Super-Humean, if one exists, will be inclined to reject all of these solutions of general relativity as "mathematical surplus" that doesn't correspond to physically possible worlds. She could thereby even count on the support of many physicists who regard CTCs and time-like (also called "naked") singularities as mere pathologies. More objectionable, to my mind, is the fact that the metaphysics I've sketched here is still a shameless escape into the Super-Humean subterfuge, an unmerited appropriation, rather than a philosophical interpretation, of general relativity. Yet, it seems much more defensible than the radical Super-Humeanism of Huggett, Esfeld and co. This shows that the main problem with their proposal is neither relationalism nor the best system account of space per se, but the extremes to which they take them in the name of parsimony.

\section{Conclusion}

Esfeld and collaborators deserve much credit for pushing the Humean project to its limits, following the principle of parsimony summarized in Frank Jackson's armchair metaphysics credo that the methodology "is not that of letting a thousand flowers bloom but rather that of making do with as meagre a diet as possible" (Jackson, 1994, p. 25, requoted in Esfeld and Deckert, 2017).

Unfortunately, the proposed "minimalist ontology" is not just meagre but starving. It is isolated from any input from empirical sciences and fails as a metaphysical foundation of space or spacetime, both as given to us by basic intuition and as described by our most successful physical theories.

Concerning the first issue, our discussion in section 2 suggests that the best system account in general requires a priori ontological commitments. It seems like an interesting challenge for Humeans how to make such commitments while credibly adhering to a methodology of naturalized metaphysics. I am not aware that any convincing answer has already been put forward.

Concerning the second issue, I have suggested how the gravest shortcomings of Super-Humeanism could be overcome by applying the regularity theory of space to relations that carry more geometric structure, namely (relative) 3-dimensional directions in addition to (relative) distances. This option, however, remains to be explored in more detail and still doesn't amount to a metaphysics that I personally endorse.

Regarding the broader philosophical discourse, I hope to have convinced the reader (if any persuading was necessary) that we must assess the empirical adequacy of a metaphysical theory by sufficiently robust standards - or else the criterion becomes trivial. The standards I propose (and discussed in section 3) are typicality and a reasonably short cognitive distance between the fundamental ontology and our manifest image of the world. By them, we recognize that while it may be true that we don't have direct empirical access to intrinsic properties, or necessary connections, or to the geometry of spacetime, renouncing all of them at once leaves us with an ontology that is too meagre to match the world that we experience.

Acknowledgements: I thank Michael Esfeld, Frida Trotter, Alastair Wilson and three anonymous referees for helpful comments and suggestions. 


\section{References}

Barbour, Julian, and Bruno Bertotti. 1982. "Mach's Principle and the Structure of Dynamical Theories."

Proceedings of the Royal Society A 382:295-306.

Belot, Gordon. 2000. "Geometry and Motion." British Journal for the Philosophy of Science 51 (4):561-595.

Bhogal, Harjit, and Zee R. Perry. 2017. "What the Humean Should Say about Entanglement." Noûs 51

(1):74-94.

Callender, Craig. 2015. “One World, One Beable." Synthese 192 (10): 3153-77.

- - - 2017. What Makes Time Special? Oxford, New York: Oxford University Press.

Cohen, Jonathan, and Craig Callender. 2009. "A Better Best System Account of Lawhood." Philosophical Studies 145 (1):1-34.

Deckert, Dirk-André, Michael Esfeld, and Andrea Oldofredi. 2016. "A Persistent Particle Ontology for QFT

in Terms of the Dirac Sea." British Journal for the Philosophy of Science, Preprint http://philsci-

archive.pitt.edu/12375/, arXiv:1608.06141[physics.hist-ph].

Esfeld, Michael. 2014. "Quantum Humeanism, or: Physicalism without Properties." The Philosophical Quarterly 64 (256):453-470.

Esfeld, Michael, and Dirk-André Deckert. 2017. A Minimalist Ontology of the Natural World. Routledge

Studies in the Philosophy of Mathematics and Physics. New York: Routledge.

Esfeld, Michael, and Vincent Lam. 2008. "Moderate Structural Realism about Space-Time." Synthese 160

(1):27-46. https://doi.org/10.1007/s11229-006-9076-2.

Esfeld, Michael, and Vincent Lam. 2011. "Ontic Structural Realism as a Metaphysics of Objects." In

Scientific Structuralism, edited by A. and P. Bokulich, 143-159. Dordrecht: Springer.

Esfeld, Michael, Dustin Lazarovici, Mario Hubert, and Detlef Dürr. 2014. "The Ontology of Bohmian

Mechanics." British Journal for the Philosophy of Science 65 (4):773-96.

Esfeld, Michael, Dustin Lazarovici, Vincent Lam, and Mario Hubert. 2017. "The Physics and Metaphysics of

Primitive Stuff." British Journal for the Philosophy of Science 68, pp. 133-161. DOI

10.1093/bjps/axv026

Hall, Ned. 2009. "Humean Reductionism about Laws of Nature." Unpublished.

Huggett, Nick. 2006. "The Regularity Account of Relational Spacetime." Mind 115 (457):41-73.

Jackson, Frank. 1994. "Armchair Metaphysics." In Philosophy in Mind. The Place of Philosophy in the Study of Mind, edited by Michaelis Michael and John O'Leary Hawthorne, 23-42. Dordrecht: Kluwer.

Lewis, David. 1973. “Causation.” Journal of Philosophy 70:556-567.

- - . 1986. Philosophical Papers. Vol. 2. Oxford: Oxford University Press.

- - . 1994. "Humean Supervenience Debugged." Mind 103 (412):473-490.

Loewer, Barry. 2007. "Laws and Natural Properties." Philosophical Topics 35 (1/2):313-28.

Maudlin, Tim. 1997. "Descrying the World in the Wave Function." The Monist 80 (1):3-23.

- - . 2007. The Metaphysics within Physics. New York: Oxford University Press.

Miller, Elizabeth. 2014. "Quantum Entanglement, Bohmian Mechanics, and Humean Supervenience."

Australasian Journal of Philosophy 92:567-583.

Saunders, Simon. 2013. "Rethinking Newton's Principia." Philosophy of Science 80 (1): 22-48.

Sellars, Wilfried. 1963: "Empiricism and the Philosophy of Mind." In Science Perception and

Reality. London: Routledge and Kegan Paul, pp. 127-96.

Vassallo, Antonio, Dirk-André Deckert, and Michael Esfeld. 2017. "Relationalism about Mechanics Based on a Minimalist Ontology of Matter." European Journal for Philosophy of Science 7, pp. 299-318. DOI 10.1007/s13194-016-0160-2

Vassallo, Antonio, and Michael Esfeld. 2016. "Leibnizian Relationalism for General Relativistic Physics."

Studies in History and Philosophy of Modern Physics 55:101-7.

Wald, Robert M. 1984. General Relativity. University of Chicago Press. 